\title{
Internal explosion. Pressure peaks
}

\author{
Vyacheslav Gorev and Evgeniya Chelekova* \\ Moscow State University of Civil Engineering, Yaroslavskoe shosse, 26, Moscow, 129337, Russia
}

\begin{abstract}
Internal emergency gas explosions occur at threatening intervals and cause significant destruction. The level of destruction indicates the imperfection of protection methods. Documents regulating the use of safety structures for the protection of buildings during an internal explosion are limited by the assignment of the area of openings covered by safety structures, without taking into account the properties of these structures, attachment methods and the rate of pressure increase during an explosion. The purpose of the work is to take into account as much as possible the influence of the properties of the protective structures, their attachment and the nature of the explosion on the dynamics of the explosion pressure change. The second goal is to obtain a methodology for converting the results of experimental results obtained on small volumes to determine the parameters of an explosion in conditions of large volume. The goals are achieved by the theory of dimensions and similarity using numerical modeling. The work revealed dimensionless complexes describing pressure dynamics both during opening of openings and at the moment of maximum power of energy release during explosion. Possibility of experimental scale modeling of processes of opening of safety structures is shown. In particular, it is shown that during an explosion in premises of a small volume (residential), the pressure during opening is more often critical.
\end{abstract}

\section{Introduction}

Emergency gas explosions in residential and industrial buildings occur regularly. This indicates a lack of preventive events.

The devastating effects of these explosions indicate the poor effectiveness of existing measures to protect construction facilities. If we talk about construction measures to protect against internal explosions, then they are reduced to volumetric planning solutions that should ensure the stability of the object as a whole in case of local losses of load capacity due to an explosion and the use of safety structures, the purpose of which is to reduce explosive loads to an acceptable level during an explosion.

\footnotetext{
*Corresponding author: SalymovaEY@mgsu.ru
} 
Acceptable level of loads is determined by permissible value of risk of various damages and calculation of load-bearing capacity of building structures under action of explosive dynamic loads. In this work we will talk about the use of safety structures to reduce the level during internal explosions. The most effective use of safety structures for these purposes in quasi-stationary explosions. These explosions are characterized by the absence of wave effects and a negligible pressure gradient in the volume. That is, you can consider the pressure to be the same at all points in the volume at every moment in time.

Such conditions are realized at relatively low combustion rates. Lightweight panels are considered as safety structures, relatively weakly attached to the protected object. It was such quasi-stationary explosions that were considered in [1-2]. [3-4] regulates the area of openings covered by safety structures. In particular, these documents state that the area of openings is determined by calculation. However, there is no methodology for such calculations. The area required to ensure safety of openings covered by safety structures also depends on the properties of the structures themselves. The main requirement for safety structures is that they must have time to open the opening so quickly that the explosive load does not exceed a critical value, that is, it does not cause a loss of load capacity of the main structures. Currently, the properties of the protective structures are not associated with the nature of the explosion. In works [1-2] dependencies of maximum explosion pressure during explosion in initially depressurized volume and in volume with closed unloading hole before explosion are obtained. This dependence is expressed only through a dimensionless expiration factor and does not depend on the properties of the protective structures and the method of their attachment to the protected volume. In works [5-6], the results [1-2] are subject to criticism in the part where we are talking about an explosion in a depressurized chamber. In particular, experimental data of works [1-2] are given as evidence of inference imperfection [5-6]. However, from our point of view, works [1-2] consider only quasi-stationary explosions, while the conditions of experiments [5-6] correspond to wave explosions. Therefore, criticism is untenable. Influence of properties of safety structures on explosion parameters was studied in works [7-9]. They show that the characteristics of the safety structures and the method of their attachment significantly affect the nature of the explosion. Experiments were carried out on installations modeling technical devices and do not take into account the features of the construction complex objects, such as volume of rooms and the attachment of safety structures, namely windows, in the depth of window openings. In [10-11], it is noted that the depth of sealing of safety structures significantly affects the nature of the explosion. It is especially important to consider this for premises of small volume - less than $100 \mathrm{~m} 3$. All experiments to study the internal quasi-stationary explosion are carried out at installations of small volume compared to the volume of real objects. However, there are no rules for applying the results of these studies to explosions at real objects. The purpose of this work is to determine the rules for transferring the results of experiments on model installations to real explosions, taking into account the scale effect and characteristics of safety structures and the method of their attachment.

\section{Material and methods}

The following is an analysis of quasi-stationary explosions for the conditions of the construction complex facilities. Such objects are not designed for high overpressure. This limitation $(\Delta \mathrm{P} \leq 30 \mathrm{kPa})$ will simplify the analysis, but does not change the principle approach and does not affect the accuracy of the conclusions. Safety structures are also 
used to protect high-strength process equipment, for example, reactors that operate at high pressures. When gas flows out of such volumes, it is necessary to use a ratio that significantly takes into account compressibility. For an internal explosion with a pre-closed opening, a typical pressure change oscillogram is shown in Fig. 1.

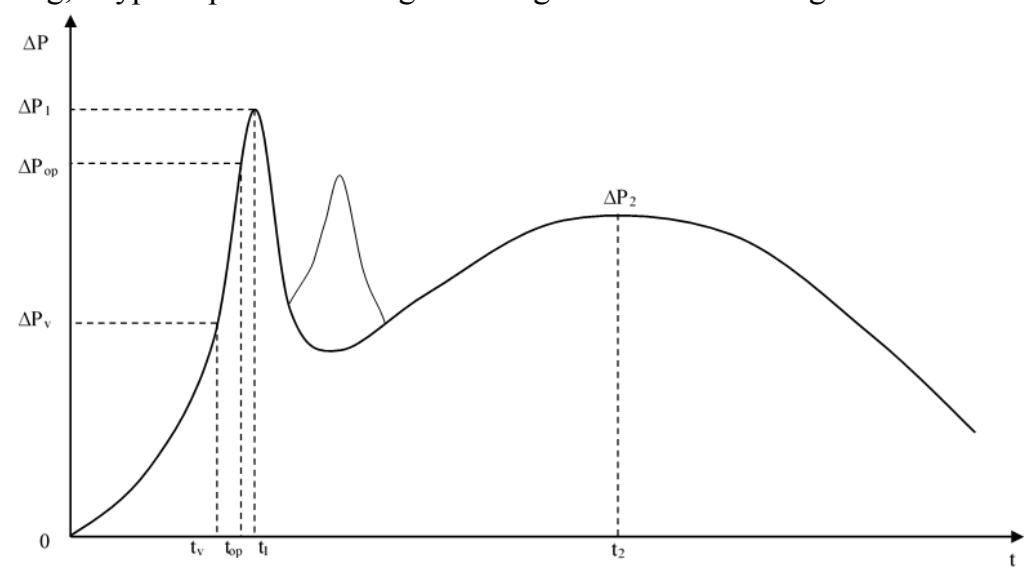

Fig.1. Typical dependence of the internal explosion pressure on the internal explosion. $\Delta \mathrm{P}_{\mathrm{v}}, \mathrm{t}_{\mathrm{v}}$ - pressure and opening time; $\Delta \mathrm{P}_{\mathrm{op}}, \mathrm{t}_{\mathrm{op}}$ - pressure and opening time of the gas outlet opening; $\Delta \mathrm{P}_{1}, \mathrm{t}_{1}$ - pressure and time of the first peak; $\Delta \mathrm{P}_{2}, \mathrm{t}_{2}$ - pressure and time of the second peak

Opening pressure $\Delta \mathrm{P}_{\mathrm{v}}$ means the pressure at which the bonds holding the safety structure are broken. After time $t_{v}$, the safety structure moves in the depth of the opening during the time $t_{0 p}-t_{v}$. During this time, the opening is still closed, and the pressure is growing like a closed volume. At time $t_{0 p}$, the safety structure begins to open the opening for the outflow of gases. After that, the pressure increase rate decreases until at the moment $\mathrm{t}_{1}$ the pressure increase stops, and the first pressure peak $\Delta \mathrm{P}_{1}$ occurs. If the safety structure is quite efficient, then the first peak comes quickly enough, and the distance traveled by the

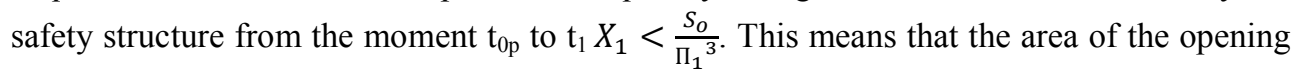
$\mathrm{S}_{0}$ is not fully open, and with the further movement of the safety structure, the pressure drops until the distance traveled by it reaches the value of $X^{*}=\frac{s_{0}}{\Pi}$; where $\Pi$ - is the perimeter of the opening. At this moment, the opening is fully open, and the explosion continues, and the pressure begins to grow again until the second peak $\Delta \mathrm{P}_{2}$.

At the second peak, the rate of emission of an additional volume of gases due to combustion is maximum and equal: the volumetric rate of outflow at this moment.

$$
\mathrm{A}_{f} U_{g}[\sigma-1]=K_{p} S_{0} \sqrt{\frac{2 \Delta P_{2}}{\rho_{x}}}
$$

Where $\mathrm{A}_{f}=\mathrm{K}_{f} V_{0}{ }^{2 / 3}$ maximum flame area at explosion, $\mathrm{m}^{2}, \mathrm{U}_{\mathrm{g}}$ - burning rate, $\mathrm{m} / \mathrm{s} ; \mathrm{V}_{0}$ is the free volume of the room, the $\mathrm{m}^{3}, \mathrm{~K}_{\mathrm{f}}$ - is a geometrical factor, $\rho_{\mathrm{x}}$ - density of the expiring gas, $\mathrm{kg} / \mathrm{m}^{3}, \rho_{x}=\rho_{o}$ at the expiration of cold mix and $\rho_{x}=\frac{P_{0}}{\sigma}, \sigma-$ - extent of expansion when burning.

Thus, in order to connect the pressure at the second peak, it is necessary to compare the complexes: 


$$
\frac{S_{0} K_{\mathrm{p}}}{U_{g}(\sigma-1) K_{f} V_{0}{ }^{2 / 3}}
$$

For equality of pressure at the second peak during experiments on a small chamber and pressure at a real large-scale explosion, it is enough to observe equality:

$$
\left(\frac{S_{0} K_{\mathrm{p}}}{U_{g}(\sigma-1) K_{f} V_{0}^{2 / 3}}\right)_{M}=\left(\frac{S_{0} K_{\mathrm{p}}}{U_{g}(\sigma-1) K_{f} V_{0}{ }^{2 / 3}}\right)_{N}
$$

Index "M" means, belonging to the explosion on the model, index "N" - to the natural size.

For the case of geometrically similar situations and mixtures with the same A, the values: $K_{f}, K_{r}$ and $\sigma$ in (3) are preserved. In this form, equation (3) coincides with the equality of dimensionless expiration coefficients in [1-2].

In order to provide a pressure link between the model experience and the actual explosion at the first peak, it is necessary to consider the explosion process from the moment it begins.

From the moment $\mathrm{t}=0$ to the moment $\mathrm{t}_{0 \mathrm{p}}$, the pressure, under the assumptions about low overpressure, varies according to the law:

$$
\Delta P(t)=\Delta P_{0 p}\left(\frac{t}{t_{0 p}}\right)^{3}
$$

Opening pressure is $\Delta \mathrm{P}_{0 \mathrm{p}}$ related to opening pressure $\Delta \mathrm{P}_{\mathrm{v}}$ :

$$
\frac{\Delta P_{0 p}}{\Delta P_{V}}=\left(\frac{t}{t_{0 p}}\right)^{3}=(1+\theta)^{3}
$$

The opening pressure is $\Delta \mathrm{P}_{\mathrm{v}}$ related to the parameters of the combustible mixture:

$$
\frac{\Delta P_{V}}{P_{0}}=\frac{4 \pi U_{g}{ }^{3} t_{V}{ }^{3}(\sigma-1) \sigma^{2} \gamma}{3 V_{0}}
$$

The value $(1+\theta)$ is related to parameter $B$, which determines the movement of the safety structure inside the opening [11-12]:

$$
\begin{aligned}
& \frac{4}{\mathrm{~B}}=\left[\frac{\left(1+\theta_{0}\right)^{5}}{5}-\left(1+\theta_{0}\right)+\frac{4}{5}\right] \\
& B=\frac{\Delta \mathrm{P}_{V}^{5 / 3} 3 V_{0}^{2 / 3}}{\rho_{\Pi} \mathrm{X}_{0} U_{g}^{2} \mathrm{P}_{0}^{2 / 3}\left(4 \pi \gamma(\sigma-1) \sigma^{2}\right)^{2 / 3}}
\end{aligned}
$$

In (7) and (8) $\rho_{\Pi}=\frac{M}{S_{0}}$ - the mass of the unit area of the safety structure in the opening. Thus, parameter B determines the dynamics of the movement of the safety structure inside the opening and the dynamics of pressure change before the start of gas outflow. After the beginning of the expiration, the pressure dynamics in the volume and the movement of the safety structure are described by the following expressions:

$$
\begin{gathered}
\frac{d \Delta \bar{P}}{d \bar{t}}=3(1+\bar{t})^{2}-\frac{2^{1 / 2} K_{p}}{\left(1+\theta_{0}\right)^{1 / 2}}\left(\frac{P_{0}}{\Delta P_{V}}\right)^{1 / 6} \frac{s_{0}}{U_{g 1} V_{0}^{2 / 3}}\left(\frac{P_{0}}{\rho_{0}}\right)^{1 / 2} \frac{\bar{X}_{1}\left(1+\Delta \bar{P}_{1}\right)^{1 / 2}}{\left(1+\theta_{0}\right)^{1 / 2}\left[\frac{4}{3} \pi \gamma \sigma^{2}(\sigma-1)\right]^{1 / 3}} \\
\Delta \bar{P}(0)=0 ; \quad \begin{array}{c}
\frac{d^{2} \overline{\mathrm{X}}_{1}}{d \bar{t}^{2}}=B(1+\theta)^{5}(1+\Delta \bar{P}) \overline{\mathrm{X}}_{0} \\
\frac{d^{2} \overline{\mathrm{X}}_{1}}{d \bar{t}} \neq \frac{B \overline{\mathrm{X}}_{0}}{4}(1+\theta)\left[(1+\theta)^{4}-1\right] \\
\bar{X}_{1}(0)=0 \\
\bar{t}=0
\end{array}
\end{gathered}
$$

In expressions (9-11) the following symbols are used:

$$
\bar{X}_{1}=\frac{X_{1} \Pi}{S_{0}} ; \bar{X}_{1}=\frac{X_{1} \Pi}{S_{0}} ; \bar{t}=\frac{t-t_{o p}}{t_{o p}} ; \Delta \bar{P}_{1}=\frac{\Delta P-\Delta P_{o p}}{\Delta P_{o p}}
$$


From the fact that the expressions are written in a dimensionless form, it follows that to equal the pressures at the first peak during the model experience and natural explosion, the following conditions must be met. The case of determining geometrically similar situations and the same degrees of expansion, in particular the same combustible mixtures, is discussed below. At the same time, the degree of expansion $\beta$ should be the same, and the burning rate $\mathrm{U}_{\mathrm{g}}$ may differ due to different explosion conditions and scale effect.

From equation (9) it is necessary to equal the values $(1+\theta)^{1 / 2}$, which is equivalent to the equality of the parameters "B" (7). For geometrically similar conditions and one combustible mixture from (9), it should also be:

$$
\left(\frac{S_{0}}{U_{g 1} \Delta P_{V}{ }^{1 / 6} V_{0}{ }^{2 / 3}}\right)_{M}=\left(\frac{S_{0}}{U_{g_{1} \Delta P_{V}}{ }^{1 / 6} V_{0}^{2 / 3}}\right)_{N}
$$

For the case of identical autopsy pressures $\Delta \mathrm{P}_{\mathrm{v}}$ the multiplier $\Delta \mathrm{P}_{\mathrm{V}}{ }^{1 / 6}$ is omitted. If the $\Delta \mathrm{P}_{\mathrm{v}}$ pressure is not established in a natural explosion, that factor can also be lowered due to a small degree of $1 / 6$ and small scatters in the opening pressure, approximate but 1.5 times.

In expressions (3) and (13), index 1 and 2 at the combustion rate $\left(\mathrm{U}_{\mathrm{g} 1}\right.$ and $\left.\mathrm{U}_{\mathrm{g} 2}\right)$ mean that the combustion rates at the peaks may not coincide in both model and real explosions. As a result, the conditions of equal pressure at the first peak under the above conditions give the criteria of equality of the first peaks during explosion at the model installation and during explosion in real conditions:

$$
\left(\frac{S_{0}}{U_{g 1} V_{0}{ }^{2 / 3}}\right)_{M}=\left(\frac{S_{0}}{U_{g 1} V_{0}^{2 / 3}}\right)_{N} ;\left(\frac{U_{g 1}{ }^{3} \rho_{\Pi}}{\Pi}\right)_{M}=\left(\frac{U_{g 1}{ }^{3} \rho_{\Pi}}{\Pi}\right)_{N} ;\left(\frac{\mathrm{X}_{0} \Pi}{S_{0}}\right)_{M}=\left(\frac{\mathrm{X}_{0} \Pi}{S_{0}}\right)_{N}
$$

If the safety structure is not buried in the opening, that is, $\mathrm{X}_{0}=0$, then the third condition in (14) falls away. The conditions of the pressure ratio at the peaks in the same explosion will be discussed below.

The pressure at the second peak can be estimated:

$$
\Delta P_{2}=\frac{1}{2}\left(\frac{\kappa_{f} V_{0}^{2 / 3}}{S_{0} K_{\mathrm{p}}}\right)^{2} \frac{U_{g}^{2} \rho_{0}(\sigma-1)^{2}}{\sigma}
$$

The last expression assumes the expiration of combustion products through the opening. This is indicated by the presence in the denominator of the value a - the degree of expansion during combustion. On the one hand, the expiration of light combustion products leads to an increase in the exhaust speeds gases through the opening, and this helps to reduce the explosion pressure. On the other hand, an increase in the rate of gas outflow through the opening causes a discharge wave that propagates inside the volume and disturbs the front of the flame.

This causes the combustion rate to increase, and hence the pressure to increase. Competition of these processes is considered in works [13-15]. The pressure at the first peak consists of an increase in pressure in the sealed volume until the opening of the openings $t_{0 p}$ and is determined from (5). After the opening of the opening begins and before the first peak occurs, the pressure increase is determined by a combination of parameters (14). In case of pressure increase in temporary section $t_{0 p}-t_{1}$ is determined either experimentally or by system solution (9-12). A numerical solution for a wide change in parameters (14) gives [16]:

$$
\Delta P_{1}=(1,1 \div 1,2) \Delta P_{o p}
$$




\section{Results}

The results of the pressure calculations at the first and second peaks and the comparison of the results of these calculations are discussed below.

Example 1.

$\mathrm{V}_{0}=10^{3} \mathrm{~m}^{3}, \mathrm{~K}_{\mathrm{f}}=4, \mathrm{~K}_{\mathrm{p}}=0.6, \mathrm{~S}_{0}=21,6 \mathrm{~m}^{2}, \mathrm{U}_{\mathrm{g} 2}=1.4 \mathrm{~m} / \mathrm{sec}$.

Pressure at second peak (15):

$\Delta P_{2}=\frac{1}{2}\left(\frac{4 * 1000^{2 / 3}}{0.6 * 21.6}\right)^{2} \frac{1.4^{2} * 1.2(7-1)^{2}}{7}=5761.3 \mathrm{~Pa}=5.8 \mathrm{kPa}$.

Параметр:

Pressure at the first peak is defined about use of additional data of $\Delta \mathrm{P}_{\mathrm{V}}=1,5 \mathrm{kPa}, \rho_{\Pi}=$ $32,4 \mathrm{~kg} / \mathrm{m}^{2}, \mathrm{X}_{0}=0.2 \mathrm{~m}, \Pi=43,2 \mathrm{~m}$.

Parameter:

$\mathrm{B}=5$, по (8), $(1+\theta)=1,67$ по (7)

$\Delta \mathrm{P}_{1}=1,5 * 1,2 * 1,67^{3}=8,38 \mathrm{kPa}$.

At change of size $\rho_{\Pi}$ up to $70 \mathrm{~kg} / \mathrm{m}^{3}$ - the extreme size allowed [3], and sizes $X_{0}$ up to $0.3 \mathrm{~m}$ turn out $\mathrm{B}=1.54,(1+\theta)=1.78$ values, $\Delta \mathrm{P}_{1}=10,15 \mathrm{kPa}$. When the value of $\rho_{\Pi}=10.8$ $\mathrm{kg} / \mathrm{m}^{3}$ decreases, and $\mathrm{X}_{0}=0.1 \mathrm{~m}$, the values of $\mathrm{B}=30,(1+\theta)=1,26, \Delta \mathrm{P}_{1}=3,6 \mathrm{kPa}$ are obtained.

It follows from this example that after determining the area of the discharge openings $\mathrm{S}_{0}$ and the pressure at the second peak $\Delta \mathrm{P}_{2}$, it is necessary to select a safety structure that provides an acceptable pressure at the first peak. This pressure can be either greater or less than the pressure at the second peak. From the analysis of the expression (14) it follows that when an explosion occurs in a smaller room, the situation at the first peak is more tense than in an explosion occurs in a larger room. This confirms the following reasoning. With volume $\mathrm{V}_{0}=10^{3} \mathrm{~m}^{3}, \mathrm{~B}=5$ and $\Delta \mathrm{P}_{1}=8.38 \mathrm{kPa}$ - the first option, with $\mathrm{V}_{0}=31.62 \mathrm{~m} 3$ (kitchen), $\mathrm{B}=0.5$ (under other similar conditions) and $\Delta \mathrm{P}_{\mathrm{op}}=10.08 \mathrm{kPa}$ and $\Delta \mathrm{P}_{1}=11.6$ $\mathrm{kPa}$, which is exactly 2 times the pressure at the second peak and 1.4 times the pressure at the first peak during an explosion in a room of $1000 \mathrm{~m}^{3}$.

Example 2.

Next, the issue of modeling an explosion in a room with a volume of $10^{3} \mathrm{~m}^{3}$ (the first version of Example 1) is considered in a chamber with a volume of $0.125 \mathrm{~m}^{3}$. From the experiment on a small chamber, $\mathrm{U}_{\mathrm{g} 1}=0.33 \mathrm{~m} / \mathrm{sec}, \mathrm{U}_{\mathrm{g} 2}=0.5 \mathrm{~m} / \mathrm{sec}$ were obtained.

From the equality of pressures at the second peak we have:

$S_{0 M}=\frac{21.6 * 0.25 * 0.5}{100 * 1.4}=0.0193 \mathrm{~m}^{2}=1.93 \mathrm{dm}^{2}$

From the equality of pressures at the first peak it follows:

$S_{0 M}=\frac{21.6 * 0.33 * 0.25}{100 * 1.4}=0.01274 \mathrm{~m}^{2}=1.27 \mathrm{dm}^{2}$

At square hole $\Pi_{\mathrm{m}}=0.4515$

From the equality of $\overline{\mathrm{X}}_{0}=\frac{\mathrm{X}_{0} \Pi}{s_{0}}$ parameters, it is obtained for the depth of sealing of the safety structure on the model plant:

$\mathrm{X}_{0 \mathrm{M}}=0,2 \frac{43,2 * 0,01274}{21,6 * 0,4515}=0,0113 \mathrm{~m}=1.13 \mathrm{sm}$

From the equality of parameters "B," it follows that the weight of the safety structure on the model installation is:

$\rho_{\text {ПМ }}=\frac{0,25 * 20 * 1,96 * 0,2}{100 * 0,0113 * 0,33^{2}} 15,94 \mathrm{~kg} / \mathrm{m}^{3}$

The mass of the model structure is: $\mathrm{M}_{\mathrm{M}}=0.203 \mathrm{~kg}$. 
As a result of Example 2, the parameters of the safety structure and the conditions of its location for simulating the explosion at the first, and second peaks were obtained. However, in this case, the simulation should be carried out in different experiments, since the requirements for the area of the open opening do not coincide due to a change in the rate of combustion at different peaks.

\section{Conclusion}

In the work on the basis of the theory of dimension and similarity, criteria were identified that allow modeling explosive loads during an internal explosion. The results apply only to quasi-stationary explosion.

Numerical modeling based on solution of system of equations (9-12) is proposed. And the possibility of large-scale experimental modeling is shown. To date, experimental results have been used as evidence of a trend in the nature of the explosion. In addition, in the opinion of the authors, the work will make it possible to conduct experimental explosions with a quantitative analysis of the results and improve the numerical models of the phenomenon under consideration.

The results obtained in this work will help in the development of model loads to describe the internal explosion. These loads can be used in calculations of building structures for stability during explosions.

\section{References}

1. D. Bradly, A. Mitcheson, I. Comb. Flame 32, 221 - 236 (1978)

2. D. Bradly, A. Mitcheson, II. Comb. Flame 32, 237 (1978).

3. Ye.Yu. Salymova, Dynamics of development of dangerous factors in buildings with enclosing structures made of sandwich panels in fires and explosions Candidate dissertation (Moscow State University of Civil Engineering, Moscow, 2015)

4. Joint venture 4.13130.2013 Fire protection systems. Restriction of distribution of the fire on subjects to protection. Requirements to space-planning and design decisions. 24.06.2013. - M.: Ministry of Regional Development of the Russian Federation, (2013).

5. D.M. Solberg. Observations of flame instabilities in large scale vented gas explosions. 18-th Sympos. (International) The combustion institute. (1980). P. $1607-1614$.

6. I.O. Moen, M. Donato Turbulent flame propagation and acceleration in the Presents of Obstacles. Progress in Astronomy and Aeronautics. V 75. (1981). p. 33 - 47.

7. V. V. Molkov, A. V. Grigorash, R. M. Eber, F. Tamanini, R. Dobashi. Vented gaseous deflagrations with inertial vent covers: State-of-the-art and progress. Process Safety Progress, 2004, vol. 23, no. 1, pp. 29-36.

8. V. V. Molkov, A. V. Grigorash, R. M. Eber, F. Vented gaseous deflagrations: Modelling of spring-loaded inertial vent covers. Fire Safety Journal, (2005), vol. 40, issue 4, pp. 307-319.

9. V. V. Molkov, A. V. Grigorash, R. M. Eber, F, D. V. Makarov. Vented gaseous deflagrations: modelling of hinged inertial vent covers. Journal of Hazardous Materials, (2004), vol. 116, issue 1-2, pp. 1-10. 
10. V. A. Gorev, V. V. Molkov. On the dependence of internal explosion parameters on the installation of safety structures in the apertures of the protecting walls of industrial and residential buildings. Pozharovzryvobezopasnost. Fire and Explosion Safety, (2018), vol. 27, no. 10, pp. 6-25.

11. Gorev V. Ensuring explosion safety of residential buildings. International Scientific Conference Environmental Science for Construction Industry - ESCI 2018, V.193, (2018), № 03046, P.8

12. V. Gorev. About dependence of parameters of internal explosion on the device of safety designs in apertures of the protecting walls of the production and residential buildings. VI International Scientific Conference "Integration, Partnership and Innovation in Construction Science and Education” (IPICSE-2018) , V. 251, 2018, 02015, p.8.

13. V.V. Molkov. Modeling the deflagratsionnykh of explosions and their consequences in objects of various gaznacheniye.//Modelling of the fires and explosions. Under a general edition of H.H. Brushlinsky and A.Ya. Korolchenko - M.: Prod. Pozhnauk, (2000). - $482 \mathrm{p}$.

14. V. V. Molkov, R. M. Eber, A. V. Grigorash, F. Tamanini, R. Dobashi. Vented gaseous deflagrations: modelling of translating inertial vent covers. Journal of Loss Prevention in the Process Industries, (2003), vol. 16, no. 5, pp. 395-402.

15. V. A. Gorev, V. V. Belyaev, V. N. Fedotov. Condition of the beginning of vibrational gas combustion in an unsealed vessel of rectangular shape. Combustion, Explosion, and Shock Waves, (1989), vol. 25, no. 1, pp. 31-34.

16. A D Korolchenko. New protecting structures on buildings of explosive production. Journal of Physics: Conference Series, V. 1425, Modelling and Methods of Structural Analysis (2019), Moscow, Russian Federation. 